\title{
A novel, minimally invasive technique to establish the animal model of spinal cord injury
}

\author{
Huiquan Duan ${ }^{1,2 \#}$, Yilin Pang ${ }^{1,2 \#}$, Chenxi Zhao ${ }^{1,2 \#}$, Tiangang Zhou ${ }^{1,2 \#}$, Chao Sun ${ }^{1,2}$, Mengfan Hou ${ }^{1,2}$, \\ Guangzhi Ning ${ }^{1,2}$, Shiqing Feng ${ }^{1,2,3,4}$
}

${ }^{1}$ Department of Orthopedics, Tianjin Medical University General Hospital, Tianjin, China; ${ }^{2}$ International Science and Technology Cooperation Base of Spinal Cord Injury, Tianjin Key Laboratory of Spine and Spinal Cord Injury, Tianjin, China; ${ }^{3}$ Department of Orthopaedics, Qilu Hospital, Shandong University, Jinan, China; ${ }^{4}$ Shandong University Center for Orthopaedics, Shandong University, Jinan, China

Contributions: (I) Conception and design: H Duan; (II) Administrative support: H Duan, Y Pang, C Zhao; (III) Provision of study materials or patients: C Sun; (IV) Collection and assembly of data: T Zhou, M Hou; (V) Data analysis and interpretation: T Zhou, C Sun; (VI) Manuscript writing: All authors; (VII) Final approval of manuscript: All authors.

\#These authors contributed equally to this work.

Correspondence to: Shiqing Feng. Department of Orthopedic Surgery, Tianjin Medical University General Hospital, 154 Anshan Road, Heping District, Tianjin 300052, China. Email: sqfeng@tmu.edu.cn.

Background: Spinal cord injury (SCI) is a traumatic disease that is associated with high morbidity, disability, and mortality worldwide. The animal spinal cord contusion model is similar to clinical SCI; therefore, this model is often used to study the pathophysiological changes and treatment strategies for humans after SCI. The present study aimed to introduce a novel, minimally invasive technique to establish an SCI model, and to evaluate its advantages compared with conventional methods.

Methods: Incision length, blood loss, length of time, and model success rate during the operation were recorded. Postoperative hematuria, incision hematoma, scoliosis [detected by micro computed tomography (Micro-CT)] and mortality were analyzed to evaluate surgical complications. The visual observation of the tissue was used to compare the effect of laminectomy by 2 methods on the scar hyperplasia at the injured site. Basso-Beattie-Bresnahan (BBB) score and catwalk automated quantitative gait analysis were conducted to measure behavioral function recovery. To evaluate the nerve function recovery of rats postoperatively, somatosensory evoked potential (SEP) and motor evoked potential (MEP) were studied by electrophysiological analyses.

Results: The results of operation-related parameters of the two models (conventional surgery group $v s$. minimally invasive surgery group) were as follows: surgical incision length: $23.58 \pm 1.58$ versus $12.67 \pm 1.50 \mathrm{~mm}$ $(\mathrm{P}<0.05)$, blood loss: $3.96 \pm 1.05$ versus $1.34 \pm 0.87 \mathrm{~mL}(\mathrm{P}<0.05)$, and total operative time: $12.67 \pm 1.78$ versus $10.33 \pm 1.92 \mathrm{~min}(\mathrm{P}<0.05)$. In addition, the success rate of the 2 models was $100 \%$. Surgical complications (conventional surgery group $v s$. minimally invasive surgery group) were as follows: hematuria: $25 \%$ versus $8.3 \%$, kyphosis: $25 \%$ versus $0 \%$, incision hematoma: $30 \%$ versus $9 \%$, and mortality: $25 \%$ versus $8.3 \%$. Micro-CT indicated severe scoliosis in the conventional surgery group. Gross tissue results showed that the conventional surgery group had more severe fibrous scar hyperplasia. The results of the BBB scores, catwalk automated quantitative gait analysis, and electrophysiology showed that the difference between the two groups was statistically significant in terms of behavioral recovery and neuroelectrophysiology.

Conclusions: The minimally invasive technique has the advantages of small incision and reduced tissue damage and surgical complications, and may be used as an alternative spinal cord contusion method.

Keywords: Spinal cord injury (SCI); animal model; minimally invasive surgery; conventional surgery

Submitted Apr 02, 2021. Accepted for publication May 17, 2021.

doi: 10.21037/atm-21-2063

View this article at: http://dx.doi.org/10.21037/atm-21-2063 


\section{Introduction}

Spinal cord injury (SCI) is a debilitating disease with no function recovery following the injury, as effective treatments are currently limited (1-5). The study of the underlying pathological mechanism and the exploration of new repair strategies of SCI are inseparable, and the SCI animal model is an important basis for experimental research. In the past few decades, various animal models have been developed to develop an understanding of the complex biomedical mechanisms of SCI and to develop therapeutic strategies for this condition (6). An ideal animal model should have several characteristics, including relevance to the pathophysiology of human SCI, reproducibility, availability, and the potential to generate various severities of injury (7).

The animal models currently used are the rat, mouse, cat, dog, pig, and primate model, with the rat model being the most frequently used. The pathological characteristics between rats and humans are similar after SCI, and large cysts are formed at the injured site. Rats not only have a clear anatomical structure of the spinal cord but relatively rare local infections and a high survival rate. In addition, rats have the following advantages such as low cost, easy care, mature function analysis technology (8). For these reasons, rats are often used for pathological studies of SCI. Because mouse genes are similar to human genes, mice are often used in genetic research (9). The spinal cord tissue of primates is closer to that of humans, so it is more suitable for SCI research. However, because of the high cost and ethical issues involved, it has not been generally used (10). The question remains as to whether large animals, such as pigs and dogs, can be used as animal models. The type (cervical and thoracic spinal cords) used in SCI research needs further verification (11). Both the cervical and thoracic spinal cords of rats can be used for SCI models. The cervical SCI model often leads to higher mortality (12). The thoracic spinal cord slices are easier to stain and observe the changes in gray matter and white matter than the cervical spine slices $(13,14)$, and the thoracic lamina bite is more convenient and easier to operate, so the SCI model mostly chooses thoracic SCI.

Different rat SCI models are usually used to explore the corresponding injury mechanisms of different SCI, which are mainly divided into contusion, transection, and crush injuries $(13,15,16)$. The animal spinal cord contusion model is similar to clinical SCI, so this model is often used in the study of pathophysiological changes and treatment strategies of humans after SCI. Research on the experimental SCI model can be traced back to 1911, when Allen used the "weight-dropping" method to construct the SCI contusion model for the first time (17). This method has the advantages of simplicity, low production cost, and easy copying, but the model also has the disadvantages of not being able to accurately determine the point of landing (18). Allen's method is the most commonly used to construct SCI animal models. Other SCI models caused by impact are also improved and optimized on this basis, and mainly include the application of the New York University (NYU)/ Multicenter Animal Spinal Cord Injury Study (MASCIS) impactor, Infinite Horizon impactor, and air gun impactor to construct animal SCI models (19-22). Regardless of which impactor is used to construct a spinal cord contusion model, the paravertebral muscles and ligaments, as well as the laminas need to be removed, which causes additional surgical damage to the animal.

However, the injury process of human SCI does not involve laminectomy and other injuries. At present, the construction of animal SCI models requires the removal of the lamina to expose the spinal cord, regardless of blower application. In the case of rats, the T9-T10 laminas are generally removed. The effect of the size of the wound and the operation method of laminectomy on the postoperative state and degree of injury of the rat is currently unknown. Therefore, the purpose of the present study was to construct an SCI model with a minimally invasive method and to evaluate its effect. We present the following article in accordance with the ARRIVE reporting checklist (available at http://dx.doi.org/10.21037/atm-21-2063).

\section{Methods}

\section{Animals}

Adult female Wistar rats weighing $210 \pm 10 \mathrm{~g}$ were purchased from the Laboratory Animal Center of the Academy of Military Medical Sciences (Beijing, China).

\section{Experimental groups}

The experimental groups were the minimally invasive surgery group and conventional surgery group, each with 12 rats according to observation parameters by simple random sampling. Huiquan Duan was aware of the group allocation at the different stages of the experiment. The rats were maintained in a temperature- and humidity-controlled 
environment with a 12:12 light-dark cycle, and allowed free access to food and water. All experiments were approved by the Ethics Committee of the Institute of Radiation Medicine, Chinese Academy of Medical Sciences \& Peking Union Medical College (approval number: IRM-DWLL-2020095), and were performed according to the National Institutes of Health in the Guide for the Care and Use of Laboratory Animals (NIH Publications No. 85-23, revised 1996).

\section{$S C I$}

The MASCIS Impactor Model III (W.M.Keck Center for Collaborative Neuroscience, Rutgers, The State University of New Jersey, USA) was applied to establish spinal cord contusion model of the two groups (23). The animals were weighed and anesthetized by isoflurane inhalation anesthesia. The skin on the dorsal side of the rat was prepared, sterilize with an iodophor cotton ball, and covered with surgical towels. A novel, minimally invasive technique was developed by our team to establish the SCI model. The thoracic vertebra 10 (T10) spinous process was located. A $0.8 \mathrm{~cm}$-long skin incision was made along the dorsal midline, and the incision length of each rat was recorded. Ophthalmic scissors were used to cut off the superficial fascia. The T10 spinous process was located again, and a scalpel was used to make a transverse incision between the T10 and T11 spinous process. The purpose was to mark the T10 spinous process and separate the ligament and muscle between the T10 and T11. Longitudinal incisions were made on both sides of the paraspinal muscles, and ophthalmic scissors were used to slightly bluntly separate them to form an H-shaped incision with the transverse incision between T10 and T11. T10 lamina was exposed after removal of the T10 spinous process and the attached muscles of the lamina by the rongeur. The laminectomy of half T10 was performed to expose the T11 cord. For the conventional surgery group, the incision length was $2.5 \mathrm{~cm}$, and the T9 and T10 laminas were exposed. Paraspinal muscles were cut about $1 \mathrm{~mm}$ wide on both sides to expose the T10-11 cords. Subsequently, contusion of the T11 cord was conducted with a height of $25 \mathrm{~mm}$. At this point, rats presented with hindlimb twitching and tail flicking, indicating that the SCI model was successfully established. Moreover, the corresponding computer software (Impactor v8.0) recorded the contusion process to further confirm whether the attack was successful. Muscles and skin were sutured. Subcutaneous saline was conducted for dehydration, and a prophylactic antibiotic was used for
7 days. Standard bladder care included daily bladder empty and Baytril for recurrent bladder infections.

\section{Record of operation-related parameters}

We recorded the incision length, blood loss, time, and model success rate during the operation. Postoperative hematuria was observed and counted during artificial urination. Postoperative incision hematoma was mainly evaluated by observation and touch, and the use of a $5-\mathrm{mL}$ syringe to decompress the hematoma achieved a good therapeutic effect. This step can also confirm the reoccurrence of hematoma. Because lamina decompression will cause instability of the spine, it is prone to kyphosis or scoliosis. Kyphosis can be observed by touch and naked eyes, and Micro-CT can be used to confirm the occurrence of kyphosis. In addition, we measured the body weight of the rats in both groups after the operation every day. The success rate of model construction and postoperative mortality of experimental animals were also observation indicators rather than exclusion criteria. Taking into account the impact of laminectomy, scar hyperplasia will occur at the exposed spinal cord segment. The visual observation of the tissue was used to compare the effect of laminectomy by 2 methods on the scar hyperplasia at the injured site.

\section{Basso-Beattie-Bresnaban (BBB) score}

To assess function recovery, behavioral tests were blindly performed from day 0 to day 42 after surgery and evaluated by BBB score. BBB score was used to evaluate the hindlimb movement for $5 \mathrm{~min}$ in an open field.

\section{Catwalk automated quantitative gait analysis}

The catwalk automated quantitative gait analysis system was used to assess the locomotor gait dynamics of the rats (Noldus Information Technology, the Netherlands). The rats were tested 6 weeks postoperatively. Each rat obtained at least 3 uninterrupted crosses training. The footprint of each animal was manually marked, and gait parameters were automatically calculated by catwalk gait analysis software (CatWalk XT 10.6). Stride length and regularity index were considered markers of hindlimb function after SCI.

\section{Electrophysiological detection}

To evaluate the nerve function recovery of rats after surgery, 

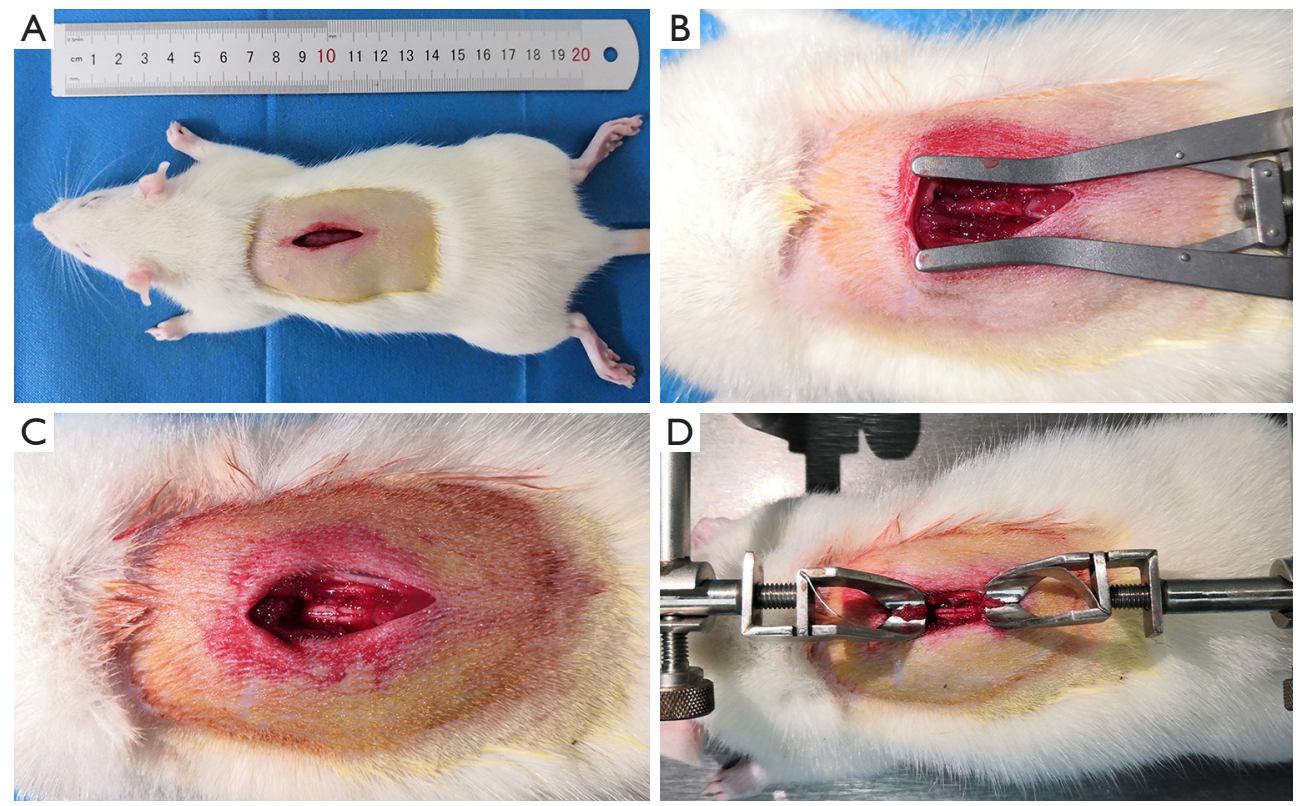

Figure 1 Conventional surgery was used to establish a spinal cord contusion model. (A) Skin incision and superficial fascia excision; (B) separation of the paraspinal muscles of T8-T11 and removal of 1-mm of paraspinal muscles from both sides; (C) T9 and T10 laminas were exposed; (D) spinous process fixation of $\mathrm{T} 8$ and $\mathrm{T} 11$.

somatosensory evoked potential (SEP) and motor evoked potential (MEP) were studied by electrophysiological analyses. Rats were anesthetized with pentobarbital, and the skin was prepared for the probe electrode, including the head and neck, between the ears, the center of the back, and the posterior sides of the hindlimbs. When measuring the SEP, the stimulating electrode was placed percutaneously in the gastrocnemius muscles of the lower limbs, the ground electrode was placed under the mid-back, and the recording and reference electrodes were placed under the skin of the head and neck between the ears. When measuring the MEP, the stimulating and reference electrodes were placed under the skin between the ears, the ground electrode was placed under the skin of the center of the back, and the recording electrode was placed percutaneously in the gastrocnemius muscle of the lower limbs. The SEP and MEP of each rat were recorded separately.

\section{Statistical analyses}

Statistical analyses were performed, and graphs were plotted using the GraphPad Prism version 8 software. Data were presented as means \pm standard deviations. Twogroup comparisons were analyzed using the unpaired $t$-test. Analysis of the differences among multiple groups or between the groups over time was done using 1-way or 2-way analysis of variance with Tukey's post-hoc test. $\mathrm{P}<0.05$ was considered statistically significant.

\section{Results}

\section{MASCIS Impactor Model III application to establish the spinal cord contusion model by conventional surgery}

The incision length was about $2.5 \mathrm{~cm}$, and the T9 and T10 lamina were exposed (Figure 1A). To expose the T1011 cords and prevent interference of the paravertebral muscles, paraspinal muscles about $1 \mathrm{~mm}$ wide on both sides should be cut off (Figure 1B,C). The spinous processes of T8 and T11 were used for rat immobilization (Figure 1D). This conventional surgical method will expose the spinal cord more thoroughly and facilitate the positioning of the impact rod, but will cause more tissue damage.

\section{Successful construction of the spinal cord contusion model by a novel, minimally invasive technique}

The length of the surgical incision and the additional tissue removal may have a certain effect on the postoperative state of the experimental rat. The current fixation of rats 

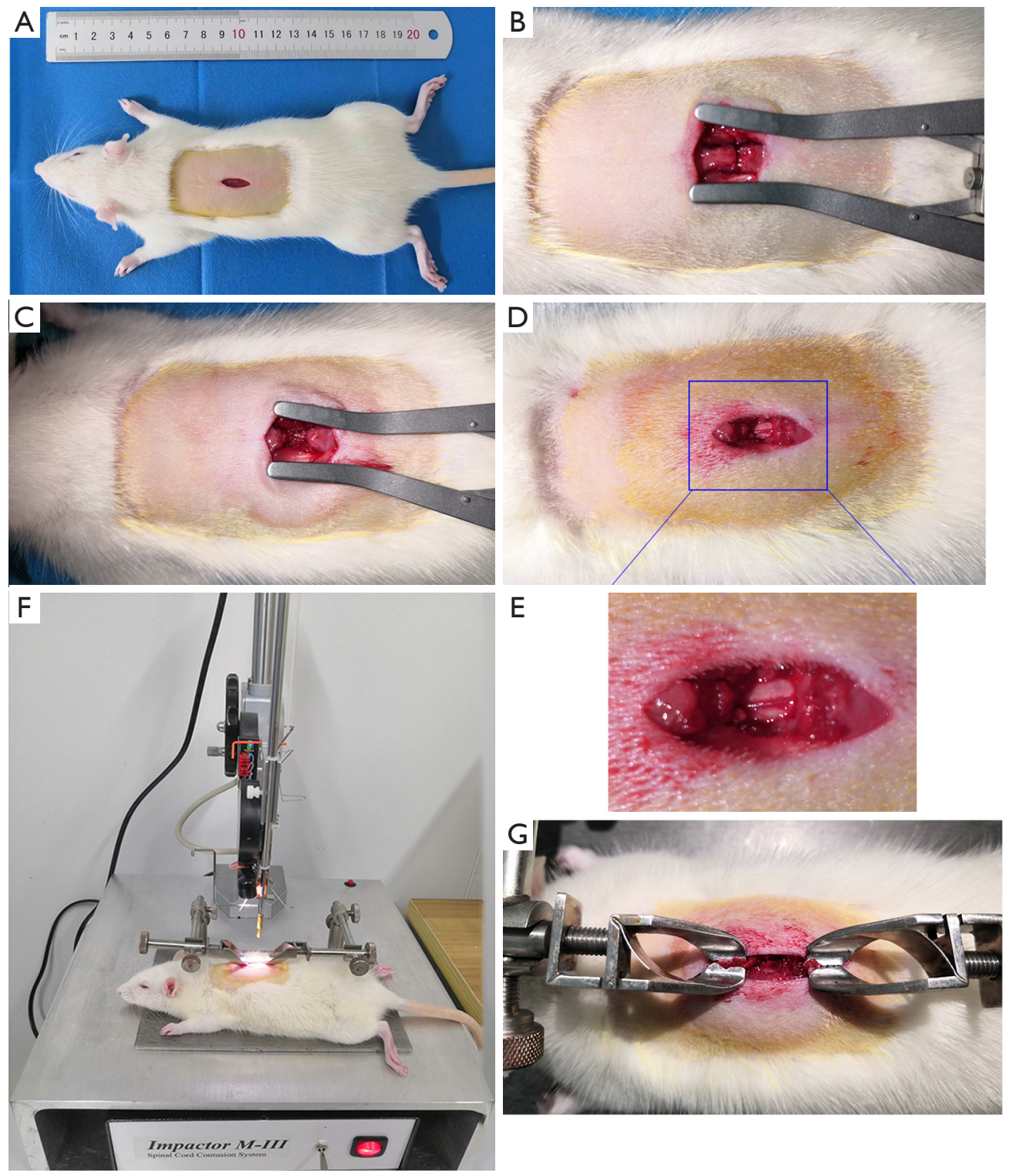

Figure 2 Novel, minimally invasive technique to establish the spinal cord contusion model. (A) Skin incision and superficial fascia excision; (B) separation of the paraspinal muscles of T9-T11, but paraspinal muscles were not removed. (C) T10 lamina was exposed; (D) laminectomy of 1/2 T10; (E) enlarged view of exposed spinal cord; (F) spinous process fixation of T9 and T11; (G) spinal cord hematoma after successful modeling.

before impact usually requires fixation of the upper and lower spinous processes, therefore, the incision should not be too small, otherwise it will affect the spinous processes fixation and surgical operation. Based on years of practice and improvement, our team shortened the surgical incision to about $0.8 \mathrm{~cm}$, and removed only the lamina of half T10, so as to achieve the successful establishment of SCI animal models. We termed this the "minimally invasive" method.
We established a contusion SCI by a novel, minimally invasive technique. After making an incision about $1 \mathrm{~cm}$ in length and following superficial fascia removal (Figure 2A), we made a transverse incision between $\mathrm{T} 10$ and $\mathrm{T} 11$ and longitudinal incisions on both sides of the paraspinal muscles, forming a $\mathrm{H}$-shaped incision (Figure $2 B$ ). Figure $2 C$ shows the spinous process of T10 removal, and T11 spinal cord exposure after the laminectomy of half T10 is shown 

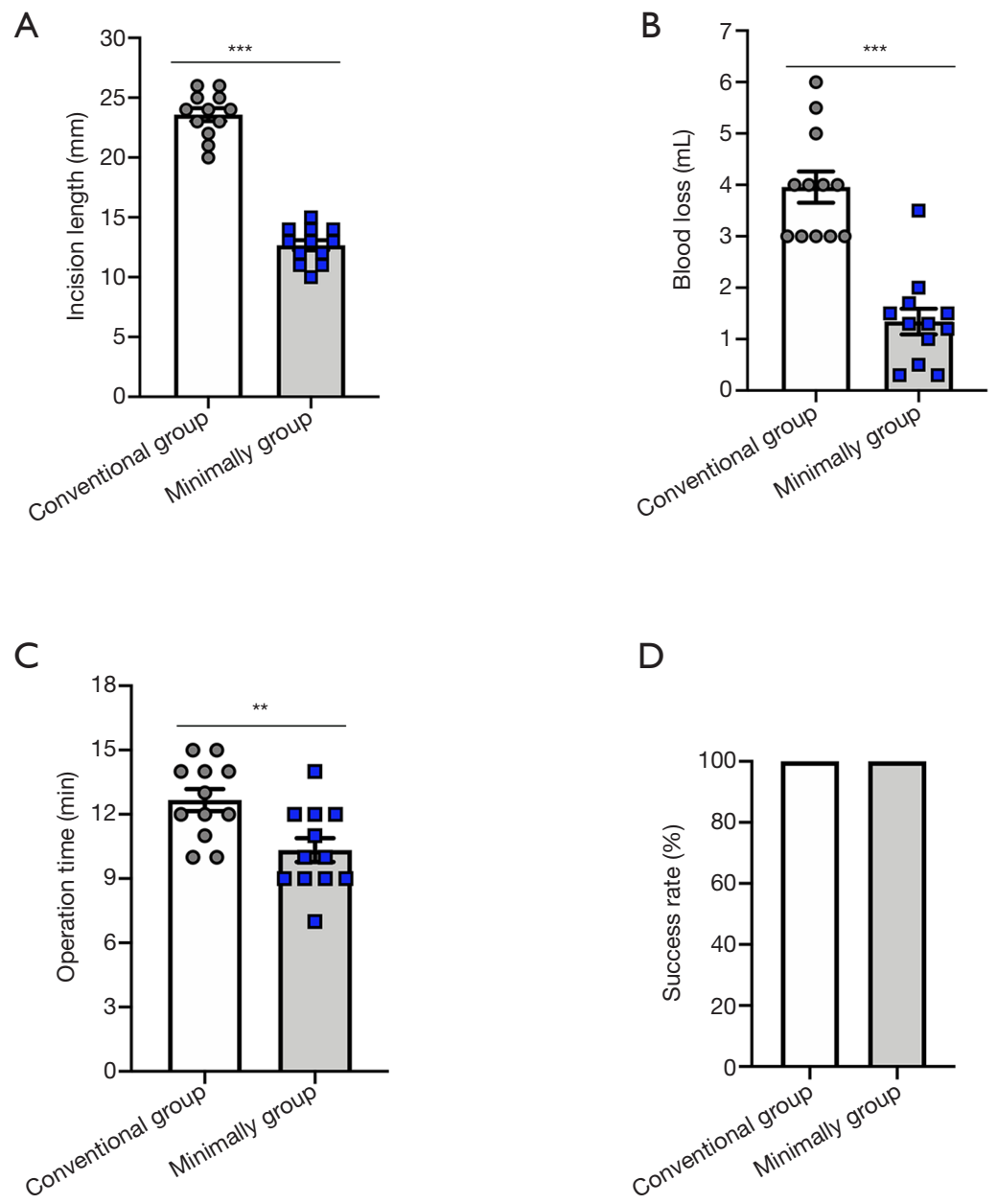

Figure 3 Operation-related parameters of the two spinal cord injury models. (A) Incision length of the conventional surgery group and minimally invasive group; (B) blood loss of the two groups; (C) operation time of the conventional surgery group and minimally invasive group; (D) model success rate of the two groups. Data were presented as means \pm standard deviations. The comparisons were analyzed using the unpaired $t$-test. ${ }^{* *}, \mathrm{P}<0.01 ;{ }^{* *}, \mathrm{P}<0.001 . \mathrm{n}=12$.

in Figure 2D and E. The MASCIS Impactor Model III was applied to establish the SCI model (Figure 2F). Figure $2 G$ shows a red hematoma on the spinal cord after impact. These results indicate that the minimally invasive technique can establish a successful spinal cord contusion model.

\section{Comparison of operation-related parameters of the two SCI models}

To compare the advantages of the minimally invasive method with previous conventional methods, we recorded the incision length, blood loss, time, and model success rate during the model construction process. The surgical incision lengths of the two models were as follows: conventional surgery group: $23.58 \pm 1.58 \mathrm{~mm}$ and minimally invasive surgery group: $12.67 \pm 1.50 \mathrm{~mm}$. Statistical analysis showed that the difference was statistically significant $(\mathrm{P}<0.001)$ (Figure 3A). The results indicated that blood loss in the minimally invasive surgery group was significantly lower than that of the conventional surgery group. Blood loss of 2 models was as follows: conventional surgery group: $3.96 \pm 1.05 \mathrm{~mL}$ and minimally invasive surgery group: $1.34 \pm 0.87 \mathrm{~mL}(\mathrm{P}<0.001)$ (Figure $3 B)$. In terms of operation time, the minimally invasive group had more advantages than the traditional group, and it took less time, thereby improving the efficiency of the model (Figure 3C). In addition, the success rate of the two models was $100 \%$ (Figure 3D), indicating that although the minimally invasive 
method required a small surgical incision and a smaller area of exposed spinal cord, it did not affect the success rate of the model.

\section{Comparison of the early complications of surgery between the 2 methods}

Stress hematuria and hematoma at the surgical incision are common postoperative complications. In addition, the removal of paravertebral muscles and laminas leads to spinal instability and increases the risk of scoliosis. We analyzed the incidence of stress hematuria, incision hematoma, and scoliosis to compare differences in early complications of surgery between the two groups. The incidence of stress hematuria in the two model methods was as follows: conventional surgery group: $25 \%$ and minimally invasive surgery group: $8.3 \%$ (Figure $4 A$ ). The incidence of kyphosis was $25 \%$ in the conventional surgery group, but no rats suffered from kyphosis in the minimally invasive surgery group (Figure 4B). The results of the incidence of incision hematoma in the two model methods were as follows: conventional surgery group: $30 \%$ and minimally invasive surgery group: $9 \%$ (Figure 4C).

We weighed the rats every day to observe the effects of the two operations. The weight of the rats in the two groups was lowest on day 2, and then began to increase. However, by days 5 and 6 , the weight decreased again and did not increase thereafter. Except for the significant difference on day 3 , there was no statistical difference in weight between the two groups (Figure 4D). In addition, our team also calculated the mortality of rats with the two model methods (time node: 28 days after surgery), and the results were as follows: conventional surgery group: $25 \%$ and minimally invasive surgery group: $8.3 \%$ (Figure $4 E$ ). Minimally invasive method mortality was found to be significantly lower than that in the conventional method group. These results indicated that the minimally invasive method has a lower incidence of early surgical complications and mortality compared with the conventional method.

\section{Comparison of late postoperative complications}

In previous experiments, we found that SCI rats were prone to developing kyphosis or scoliosis. In the present study, we considered whether the minimally invasive method would reduce the occurrence of scoliosis, because this method only removes half of the lamina. Micro-CT was conducted to observe scoliosis or kyphosis in rats. The findings indicated that obvious scoliosis occurred in the rats in the conventional surgery group (Figure $5 A, B, C$ ), while the spine shape of the rats in minimally invasive group was close to that of the normal group, and there was no visible scoliosis (Figure 5D,E,F). This result indicated that excessive removal of paravertebral muscles and laminas can cause instability and scoliosis of the spine.

Spinal cord tissues are often prepared for pathological slices, and the morphology of the tissue after sampling from rats will affect the quality and esthetics of the images after staining and photography. Because of the exposure of spinal dura after laminectomy, a large number of fibrous scars will grow into and adhere to dura. It is easy to damage or even break the spinal cord due to the traction of the scar. Therefore, we observed the proliferation of epidural fibrous scars of the above two model methods and compared them with the general morphology of the tissue. The results indicated that more fibrous scars adhered to the spinal cord in the conventional surgery group compared with the minimally invasive group (Figure $5 G$ ), and the scars adhered firmly, unless they were cut off with ophthalmic scissors. However, the scars in the minimally invasive group were smaller.

\section{Evaluation of function recovery of rats with the two model methods}

To compare the effects of the two model methods on the behavioral recovery of SCI rats, BBB score and catwalk automated quantitative gait analysis were evaluated. The results of BBB scores indicated that the two model methods had no difference in behavioral recovery (Figure 6A). Catwalk automated quantitative gait analysis showed that the difference between the two groups was not statistically significant (Figure 6B,C,D). This indicated that the size of the exposed area of the spinal cord had no visible difference in the degree of damage to rats. To further confirm sensory and motor function improvement, electrophysiological analysis was performed postoperatively (Figure 6E). There was no significant difference in the amplitude and latency of SEP and MEP between the conventional surgery group and minimally invasive group (Figure $6 F, G, H, I$ ). This further confirms that the minimally invasive surgery model is uniform and stable, and has the same degree of damage as the conventional injury group. The conventional method is also stable, as long as the spinal cord is not accidentally damaged during laminectomy. 
A

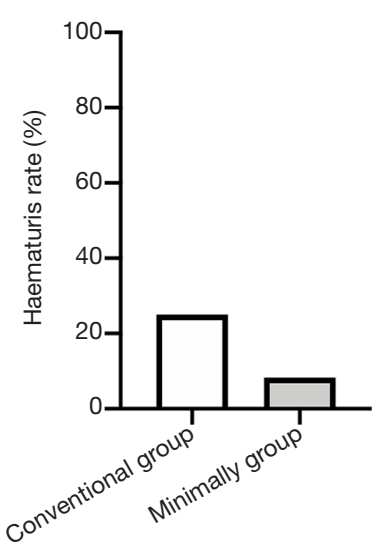

B

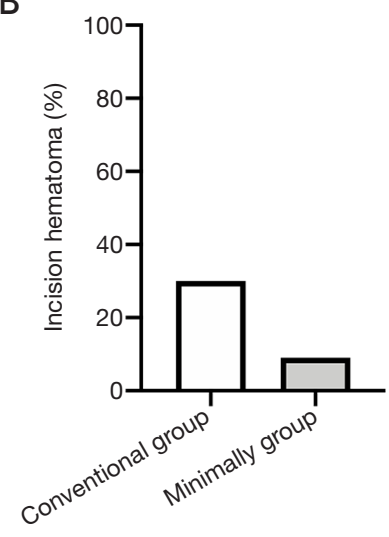

C

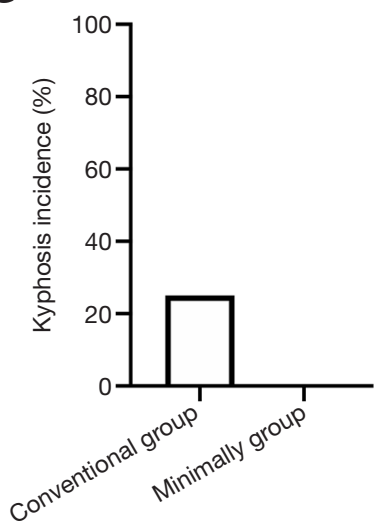

E

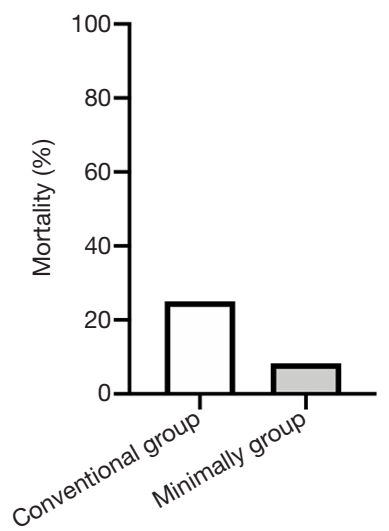

Figure 4 Early complications of surgery between the two methods. (A) Hematuria rate of conventional surgery group and minimally invasive group; (B) hematoma in the two groups; (C) incidence of kyphosis; (D) daily weight of rats to observe the effects of the two operations; (E) mortality of rats with the two model methods. Data were presented as means \pm standard deviations. The comparisons were analyzed using the unpaired $t$-test. *, $\mathrm{P}<0.05$. $\mathrm{n}=12$.

\section{Discussion}

In the present study, we introduced a novel, minimally invasive technique to establish an animal model of SCI and to evaluate its advantages compared with conventional methods. We compared operation-related parameters, early complications of surgery, late postoperative complications, and behavioral recovery after SCI. The results showed that the standard minimally invasive technique has the advantages of a small surgical incision and reduced tissue damage and surgical complications, and may be used as an alternative spinal cord contusion method.
Contusion is caused by a physical transient impact on the spinal cord; therefore, contusion model is clinically relevant. There are currently three types of devices that can produce SCI in the animal model. These are as follows: weight dropping, electromagnetic impactor, and the air gun. The weight-dropping method was first used by Allen to construct the SCI model. The NYU impactor was then introduced by Gruner in about 1992. The original NYU impactor included a metal rod weighing $10 \mathrm{~g}$, when it was dropped on the exposed spinal cord tissue to induce SCI. The NYU impactor allowed induction of a defined 

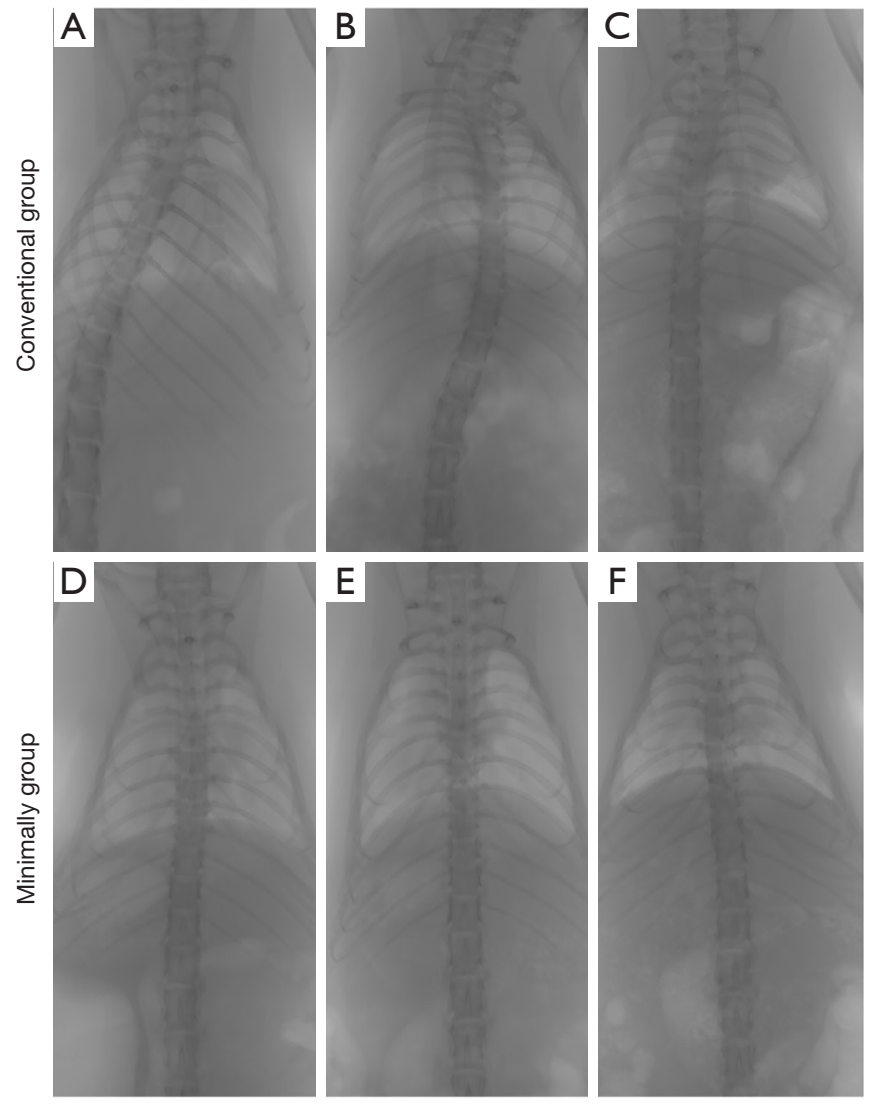

E

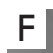

Conventional group

Minimally group

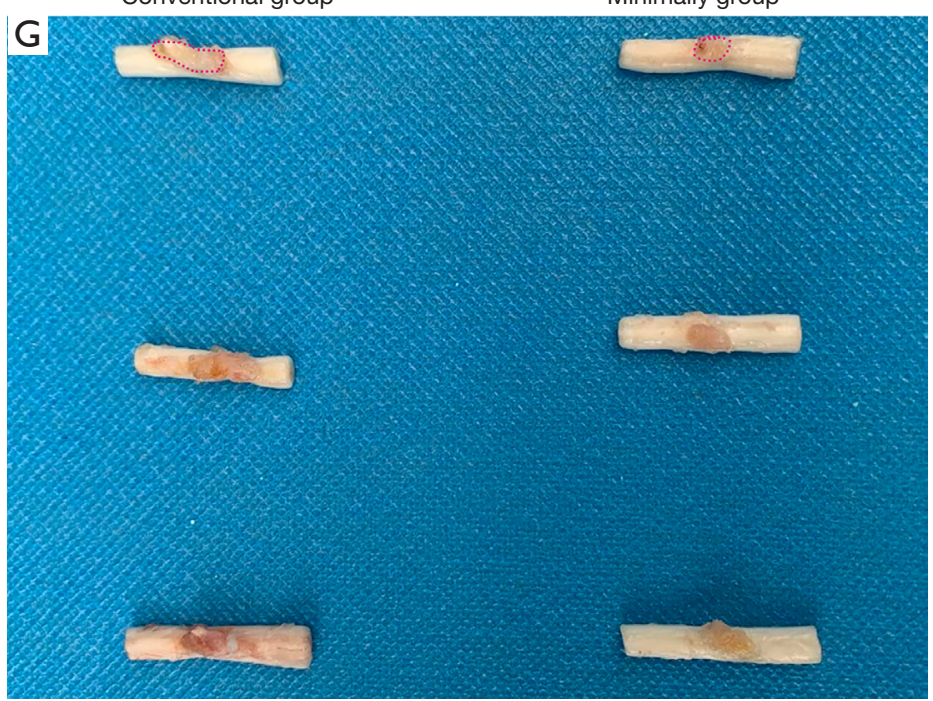

Figure 5 Comparison of the late postoperative complications between the two groups. (A,B,C) Micro-computed tomography was used to observe scoliosis or kyphosis in rats in conventional group; (D,E,F) micro-computed tomography was used to observe scoliosis or kyphosis in rats in minimally group; $(\mathrm{G})$ the visual observation of the tissue was used to compare the effect of laminectomy by 2 methods on the scar hyperplasia at the injured site. 
A

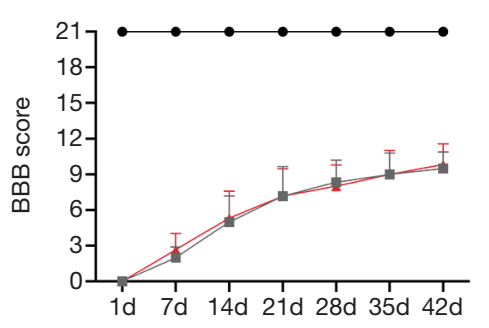

- Normal

- - Conventional group

$\star$ Minimally group
B

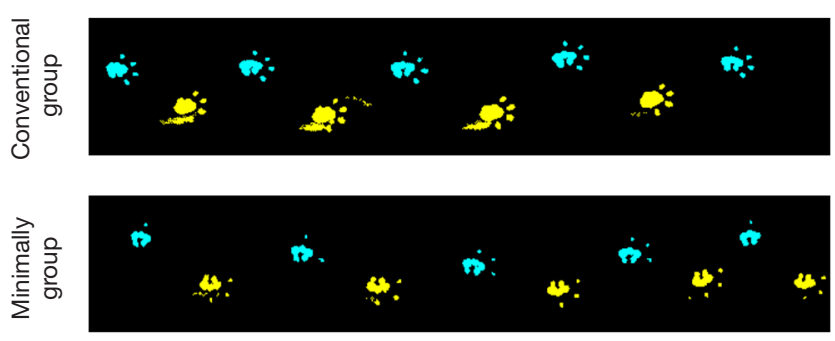

$E$

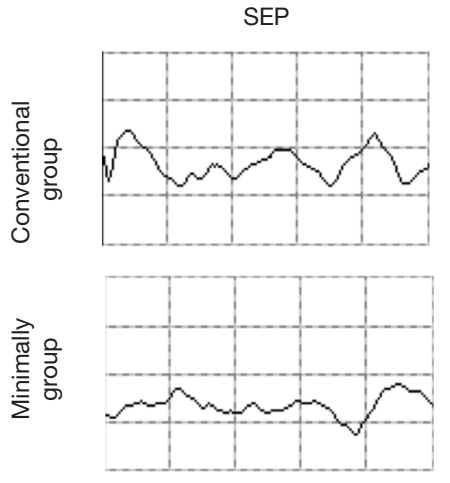

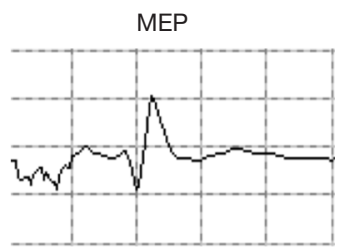

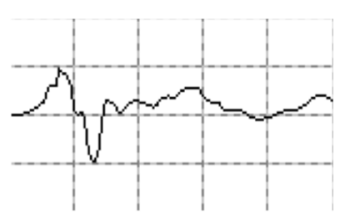

C

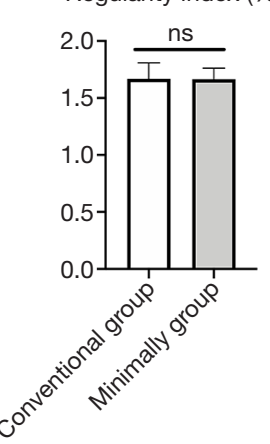

Print width (cm)

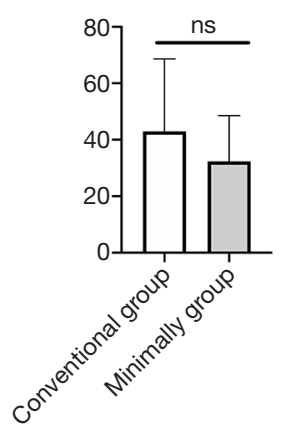

$\mathrm{H}$ SEP-Amplitude

MEP-Amplitude
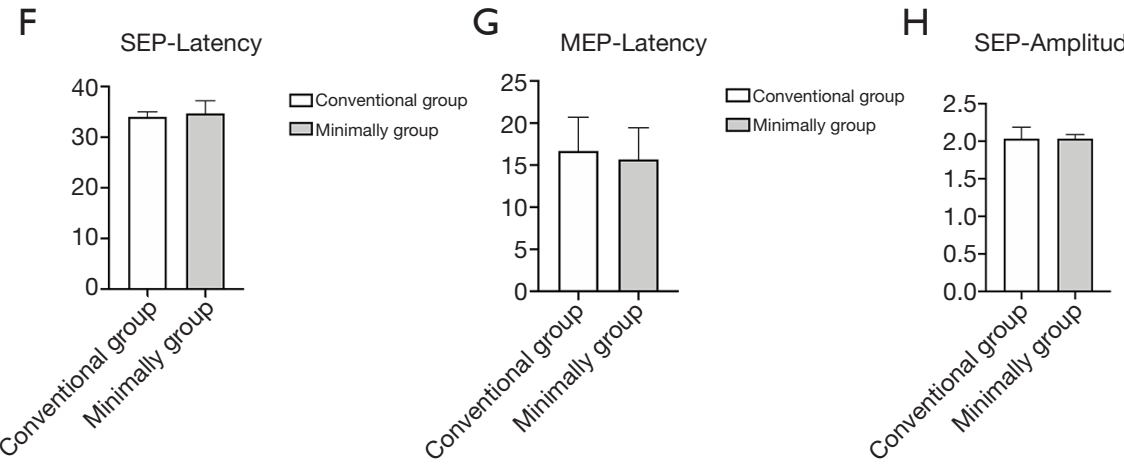

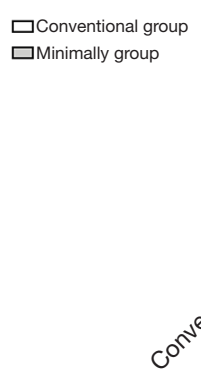

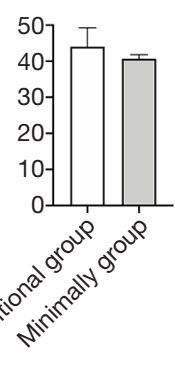

$\square$ Conventional group $\square$ Minimally group

Figure 6 Function recovery of rats with the 2 model methods. (A) Basso-Beattie-Bresnahan score; data were presented as means \pm standard deviations. Analysis of the differences among three groups was done using 2-way analysis of variance with Tukey's post-hoc test. n=6; (B) catwalk automated quantitative gait analysis of minimally invasive technique and conventional method; (C,D) stride length and regularity index of catwalk; data were presented as means \pm standard deviations. The comparisons were analyzed using the unpaired $t$-test. $\mathrm{n}=3$; (E) somatosensory evoked potential (SEP) and motor evoked potential (MEP); (F,G) quantification of latency (ms) of SEP and MEP. (H,I) quantification of amplitude $(\mathrm{uV})$ of SEP and MEP. Data were presented as means \pm standard deviations. The comparisons were analyzed using the unpaired $t$-test. $\mathrm{n}=3$.

severity of SCI by adjusting the impact height. The NYU impactor was renamed to the MASCIS impactor. Parameters, including height, velocity, and biomechanical response of the spinal cord, can be recorded for analysis or verification. The MASCIS impactor has been updated twice, and the most recent version is the MASCIS III. The
MASCIS III was first introduced in 2012 and included both electromagnetic control function and digital recording of the impact-related parameters. The MASCIS Impactor Model is now widely used worldwide, as it can establish a standardized SCI model. However, due to the special structure of the MASCIS Impactor Model III, it is 
important to fully expose the spinal cord to facilitate the precise positioning of the impact rod. The T9 and T10 laminas should be removed to expose the T10-11 cords, and at least $1-\mathrm{mm}$ of paraspinal muscles on both sides should be removed to prevent the interference of paraspinal muscles on the impact rod. The spinous processes of T8 and T11 are used for rat immobilization; therefore, the incision will be long. After several years of practice and improvement, our team gradually shortened the surgical incision to about $0.8 \mathrm{~cm}$. In addition, our team found that the monitoring electrode does not have to be in contact with the spinal cord; it can also perform its function when it contacts the paraspinal muscles or lamina. Therefore, we can remove only half $\mathrm{T} 10$ of the lamina, which can achieve the successful establishment of an SCI animal model, that is, the minimally invasive method.

To evaluate the advantages of the minimally invasive method compared with previous conventional methods, we recorded the incision length, blood loss, time, and model success rate during the model construction process. The results showed that the minimally invasive method required a shorter incision length than that of the conventional group. During the operation, we found that the shorter incision length did not affect the surgery operation and spinous processes fixation. Blood loss of the minimally invasive surgery group was significantly lower than that of the conventional surgery group. The conventional surgery group had more bleeding due to greater tissue damage, with an average bleeding volume of $3.96 \pm 1.05 \mathrm{~mL}$. According to the formula of related research, the blood volume of a rat $(200 \mathrm{~g})$ is about $11.68-14.08 \mathrm{~mL}$ (24). Blood loss of the conventional surgery group was about one-third of the blood volume of the rat, which was likely to affect the postoperative state of the rat. However, blood loss of the minimally invasive group was nearly one-sixth of the blood volume of the rat. The minimally invasive group had a shorter operation time because the exposed lamina was smaller, thereby improving the efficiency of the model. In addition, the success rate of the two models was $100 \%$, indicating that although the minimally invasive method required only a small surgical incision and a smaller area of exposed spinal cord, it did not affect the success rate of the model.

Stress hematuria and hematoma at the surgical incision are common postoperative complications. In addition, the removal of paravertebral muscles and laminas leads to spinal instability and increases the risk of scoliosis. The incidence of stress hematuria in the conventional surgery group was
$25 \%$; however, it was $8.3 \%$ in the minimally invasive group. The incidence of kyphosis was $25 \%$ in the conventional surgery group, but no rat suffered from kyphosis in the minimally invasive group. The results of the incidence of incision hematoma in the two models were $30 \%$ for the conventional surgery group versus $9 \%$ for the minimally invasive group. We recorded the mortality of rats in the two groups. Roy et al. demonstrated that the mortality of SCI experimental animal models within 1 week can be as high as $50-70 \%(25)$. In the present study, we reported the mortality rate in the conventional surgery group was $25 \%$, and it was only $8.3 \%$ in the minimally invasive group. These results indicated that the minimally invasive method has a lower incidence of early surgical complications. We weighed the rats every day to observe the effects of the two operations. The weight of the rats in both groups was lowest at day 2 and then began to increase, but by days 5 and 6 , the weight tended to decrease again, and then gradually increase thereafter. With the exception of the statistical difference on the day 3 , there was no statistical difference in weight between the rats in the two groups. We found that the reason for the postoperative weight loss in rats was due to the reduction in food and water intake, while the slow weight loss in the conventional surgery group was due to greater surgical trauma, severe disturbance to the spine and nerve roots, and bladder and gastrointestinal emptying disorders. For late postoperative complications, micro-CT was used to observe scoliosis or kyphosis in the rats. The results showed that obvious scoliosis occurred in the rats in the conventional surgery group, while the spine shape of the minimally invasive group was close to the normal group, and there was no visible scoliosis. This indicated that the excessive removal of paravertebral muscles and lamina can cause instability and scoliosis of the spine. Because of the exposure of the spinal dura after laminectomy, a large number of fibrous scars will grow into and adhere to dura. It is easy to damage or even break the spinal cord due to the traction of the scar. Therefore, we observed the proliferation of epidural fibrous scars of the above two models and compared them with general tissue morphology. The results showed that more fibrous scars adhered to the spinal cord in the conventional surgery group than in the minimally invasive group, and the scars adhered firmly, unless they were cut off with ophthalmic scissors. However, the scars in the minimally invasive group were smaller. These results indicated that the minimally invasive method can reduce the formation of fibrous scars.

BBB score and catwalk automated quantitative gait 
analysis were conducted to evaluate the effects of the two models on the behavioral recovery of SCI in rats. The results of the BBB scores and catwalk automated quantitative gait analysis showed that the two models had no difference in behavioral recovery. To further confirm sensory and motor function improvements, electrophysiological analysis was performed postoperatively. There was no significant difference in the amplitude and latency of SEP and MEP between the two groups. This further confirms that the minimally invasive model is uniform and stable, and can achieve the same degree of damage as the conventional operation method. The conventional method is also stable, as long as the spinal cord is not accidentally damaged during the laminectomy. However, the limitation of this study is that because it compares the related parameters of two kinds of surgery, the difference of the surgical procedure is bound to be an unavoidable confounder.

\section{Conclusions}

The minimally invasive method of constructing SCI has certain advantages compared with the conventional model method, including operation-related parameters, early complications of surgery, and late postoperative complications. The minimally invasive model will not affect the degree of injury and the postoperative recovery process of SCI in rats; therefore, it can be used as a novel invasive surgery to establish a stable contusion model.

\section{Acknowledgments}

Funding: The present study was supported by the National Natural Science of China (Project No. 81930070, 81772342), Tianjin Key Research and Development PlanKey Projects for Science and Technology Support (No. 19YFZCSY00660), and the Key Program of Natural Science Foundation of Tianjin (No. 19JCZDJC36300).

\section{Footnote}

Reporting Checklist: The authors have completed the ARRIVE reporting checklist. Available at http://dx.doi. org/10.21037/atm-21-2063

Data Sharing Statement: Available at http://dx.doi. org/10.21037/atm-21-2063

Conflicts of Interest: All authors have completed the ICMJE uniform disclosure form (available at http://dx.doi. org/10.21037/atm-21-2063). The authors have no conflicts of interest to declare.

Ethical Statement: The authors are accountable for all aspects of the work in ensuring that questions related to the accuracy or integrity of any part of the work are appropriately investigated and resolved. All experiments were approved by the Ethics Committee of the Institute of Radiation Medicine, Chinese Academy of Medical Sciences \& Peking Union Medical College (approval number: IRMDWLL-2020095), and were performed according to the National Institutes of Health in the Guide for the Care and Use of Laboratory Animals (NIH Publications No. 85-23, revised 1996).

Open Access Statement: This is an Open Access article distributed in accordance with the Creative Commons Attribution-NonCommercial-NoDerivs 4.0 International License (CC BY-NC-ND 4.0), which permits the noncommercial replication and distribution of the article with the strict proviso that no changes or edits are made and the original work is properly cited (including links to both the formal publication through the relevant DOI and the license). See: https://creativecommons.org/licenses/by-nc-nd/4.0/.

\section{References}

1. Guo S, Perets N, Betzer O, et al. Intranasal Delivery of Mesenchymal Stem Cell Derived Exosomes Loaded with Phosphatase and Tensin Homolog siRNA Repairs Complete Spinal Cord Injury. ACS Nano 2019;13:10015-28.

2. Formento E, Minassian K, Wagner F, et al. Electrical spinal cord stimulation must preserve proprioception to enable locomotion in humans with spinal cord injury. Nat Neurosci 2018;21:1728-41.

3. Huang H, Young W, Skaper S, et al. Clinical Neurorestorative Therapeutic Guidelines for Spinal Cord Injury (IANR/CANR version 2019). J Orthop Translat 2019;20:14-24.

4. Kang Y, Ding H, Zhou H, et al. Epidemiology of worldwide spinal cord injury: a literature review. Journal of Neurorestoratology 2018;6:1-9.

5. Zhang Z, Wang F, Song M. The cell repair research of spinal cord injury: a review of cell transplantation to treat spinal cord injury. Journal of Neurorestoratology 2019;7:55-62. 
6. Fan B, Wei Z, Yao X, et al. Microenvironment Imbalance of Spinal Cord Injury. Cell Transplant 2018;27:853-66.

7. Nardone R, Florea C, Holler Y, et al. Rodent, large animal and non-human primate models of spinal cord injury. Zoology (Jena) 2017;123:101-14.

8. Shi P, Fang Y, Yu H. Bladder response to acute sacral neuromodulation while treating rats in different phases of complete spinal cord injury: a preliminary study. Int Braz J Urol 2015;41:1194-201.

9. Muñoz-Galdeano T, Reigada D, Del Aguila A, et al. Cell Specific Changes of Autophagy in a Mouse Model of Contusive Spinal Cord Injury. Front Cell Neurosci 2018;12:164.

10. Kwon BK, Streijger F, Hill CE, et al. Large animal and primate models of spinal cord injury for the testing of novel therapies. Exp Neurol 2015;269:154-68.

11. Mazensky D, Flesarova S, Sulla I. Arterial Blood Supply to the Spinal Cord in Animal Models of Spinal Cord Injury. A Review. Anat Rec (Hoboken) 2017;300:2091-106.

12. Li K, Nicaise C, Sannie D, et al. Overexpression of the astrocyte glutamate transporter GLT1 exacerbates phrenic motor neuron degeneration, diaphragm compromise, and forelimb motor dysfunction following cervical contusion spinal cord injury. J Neurosci 2014;34:7622-38.

13. Cheriyan T, Ryan DJ, Weinreb JH, et al. Spinal cord injury models: a review. Spinal Cord 2014;52:588-95.

14. Martin JH. Harnessing neural activity to promote repair of the damaged corticospinal system after spinal cord injury. Neural Regen Res 2016;11:1389-91.

15. Rivlin AS, Tator $\mathrm{CH}$. Effect of duration of acute spinal cord compression in a new acute cord injury model in the rat. Surg Neurol 1978;10:38-43.

16. Joshi M, Fehlings MG. Development and characterization of a novel, graded model of clip compressive spinal cord

Cite this article as: Duan H, Pang Y, Zhao C, Zhou T, Sun C, Hou M, Ning G, Feng S. A novel, minimally invasive technique to establish the animal model of spinal cord injury. Ann Transl Med 2021;9(10):881. doi: 10.21037/atm-21-2063 injury in the mouse: Part 2. Quantitative neuroanatomical assessment and analysis of the relationships between axonal tracts, residual tissue, and locomotor recovery. J Neurotrauma 2002;19:191-203.

17. Allen AR. Surgery of experimental lesion of spinal cord equivalent to crush injury of fracture dislocation of spinal column: A preliminary report. J Am Med Assoc 1911;LVII:878-80.

18. Chung WH, Lee JH, Chung DJ, et al. Improved rat spinal cord injury model using spinal cord compression by percutaneous method. J Vet Sci 2013;14:329-35.

19. Scheff SW, Rabchevsky AG, Fugaccia I, et al. Experimental modeling of spinal cord injury: characterization of a force-defined injury device. J Neurotrauma 2003;20:179-93.

20. Streijger F, Beernink TM, Lee JH, et al. Characterization of a cervical spinal cord hemicontusion injury in mice using the infinite horizon impactor. J Neurotrauma 2013;30:869-83.

21. Dunham KA, Siriphorn A, Chompoopong S, et al. Characterization of a graded cervical hemicontusion spinal cord injury model in adult male rats. J Neurotrauma 2010;27:2091-106.

22. Marcol W, Slusarczyk W, Gzik M, et al. Air gun impactor-a novel model of graded white matter spinal cord injury in rodents. J Reconstr Microsurg 2012;28:561-8.

23. Young W. Spinal cord contusion models. Prog Brain Res 2002;137:231-55.

24. Lee HB, Blaufox MD. Blood volume in the rat. J Nucl Med 1985;26:72-6.

25. Roy FD, Yang JF, Gorassini MA. Afferent regulation of leg motor cortex excitability after incomplete spinal cord injury. J Neurophysiol 2010;103:2222-33.

(English Language Editor: R. Scott) 